\title{
PROKLA-Redaktion
}

\section{Editorial: Marx!}

Mit der Finanzkrise von 2008 und der Wirtschaftskrise, die 2009 folgte, wuchs geradezu schlagartig das öffentliche Interesse an Marx. Nachdem es an den ökonomischen genauso wie an den meisten sozialwissenschaftlichen Fachbereichen deutscher Universitäten schon lange keine Lehrveranstaltungen mehr zur Marxschen Theorie gegeben hatte, nachdem man in den Medien des Mainstreams Marx seit Jahren nur dann noch hervorkramte, wenn man ihm aufs Neue bescheinigen wollte, dass er überholt sei, war dieses erneute Interesse zwar bemerkenswert, aber bei näherem Hinsehen doch nicht so ganz überraschend. Während die herrschende neoklassische Theorie seit Jahrzehnten das Hohelied effizienter Märkte sang, die - wenn sie nur ausreichend dereguliert und flexibilisiert seien - nie und nimmer eine Krise hervorbringen würden, war die Krise plötzlich wieder da. Und die Regierungen, denen die Glaubenssätze des Neoliberalismus längst als unbestreitbarer common sense galten, griffen $\mathrm{zu}$ interventionistischen Maßnahmen bis hin zur Bankenverstaatlichung, um wenigstens die unmittelbaren Krisenfolgen abzumildern, waren aber genauso wie die Vielzahl der sie beratenden Experten unsicher, ob das dicke Ende nicht erst noch bevorsteht. In dieser Situation konnte sich einem schon die Frage aufdrängen, ob der alte Mann mit dem Rauschebart nicht vielleicht doch recht gehabt hatte, mit seiner Diagnose, dass Krisen keine Betriebsun- fälle, sondern notwendige Begleiterscheinungen des Kapitalismus seien? Und wenn er schon mit dieser Diagnose recht behalten hatte, sollte dann vielleicht auch am Rest seiner Kapitalismuskritik etwas dran sein? Bei einem Großteil der medialen Auseinandersetzung mit Marx konnte man den Eindruck gewinnen, dass es vor allem darum ging einen solchen Verdacht auszuräumen: Ja, mit der Krise habe Marx vielleicht einen Punkt erwischt, aber trotzdem könne man mit der Marxschen Theorie insgesamt heute nicht mehr viel anfangen - so der Tenor vieler Beiträge. Warum man aber mit der schon mehr als einmal an der Wirklichkeit gescheiterten neoklassischen Theorie, deren Vertreter in den letzten 40 Jahren so viele Nobelpreise abgeräumt hatten und die nach wie vor den Standardlehrstoff an den wirtschaftswissenschaftlichen Fachbereichen ausmacht, besser fahren würde, konnte einem niemand erklären. In so manchen studentischen und auch in einigen gewerkschaftlichen Gruppen wurden die Fragen nach der Erklärungskraft der Marxschen Theorie dagegen zum Anlass für eine vertiefte Auseinandersetzung mit Marx genommen. Kapital-Lektüre Kurse (in der Regel fernab jeder institutionellen Anbindung) hatten eine Zeitlang Hochkonjunktur und existieren zum Teil auch jetzt noch.

Mit dem vorliegenden Heft der PROKLA wollen wir Marx allerdings nicht als Krisentheoretiker diskutieren, dies erfolgt bereits in einer ganzen Reihe von Bü- 
chern und Artikeln, sondern eher nach den Potentialen seiner Theorie jenseits der Kritik der politischen Ökonomie im engeren Sinne fragen. So wichtig die Kritik der politischen Ökonomie auch sein mag, sie bildet nur einen Teil eines umfassenderen, herrschaftskritischen Projektes, das eine ökonomietheoretische Engführung nicht verträgt. Eine auf Emanzipation abzielende Gesellschaftstheorie muss weiter gehen. Nach unserem Schwerpunktheft Gesellschaftstheorie nach Marx und Foucault (PROKLA 151, Juni 2008) will die PROKLA mit dieser Ausgabe einen weiteren Beitrag dazu leisten, weitere Theorieschwerpunkte werden im nächsten Jahr folgen.

Im vorliegenden Heft diskutiert Alex Demirović zunächst das Verhältnis von Handlung und Struktur. Dabei wendet er sich gegen eine verkürzte soziologische Auffassung dieser Problematik und versucht insbesondere das Wertgesetz als Logik einer kollektiven Praxis zu reformulieren.

Urs Marti untersucht Marx als politischen Denker. Nicht selten wird bestritten, dass Marx überhaupt etwas zur politischen Philosophie beigetragen hat. Demgegenüber wird in Auseinandersetzung mit Kant, Tocqueville, Rawls und Habermas aufgezeigt, dass Marx Erhebliches zum Verständnis der Widersprüchlichkeit des liberalen politischen Denkens beigesteuert hat.

Mit neueren gesellschaftstheoretischen Ansätzen von Axel Honneth und Ulrich Beck, die „Paradoxie“ und „Ambivalenz“ als Schlüsselbegriffe einführten, setzen sich Jörg Stadlinger und Dieter Sauer auseinander. Sie machen die theoretisch fragwürdigen Grundlagen dieser Konzepte deutlich und konfrontieren sie mit Marx' dialektischem Freiheitsbegriff.

Dem oft wiederholten Vorwurf, dass Marx und Engels ökologische Problematiken und insbesondere die Bedeutung der Thermodynamik in ihren Untersuchungen ignoriert hätten, begegnen Paul Burkett und John Bellamy Foster mit einer breit angelegten Untersuchung. Sie zeigen nicht nur auf, welche Autoren und welche ökologisch relevanten Themen Marx und Engels rezipierten, sie machen auch deutlich, dass diese Themen in ihren Analysen in vielfältiger Weise anwesend sind.

Hanno Pahl setzt sich mit der schon von Marx angeschnittenen Frage einer Wissenssoziologie der Wirtschaftswissenschaften auseinander. Er diskutiert neuere Beiträge zur Debatte und fragt, inwiefern sie für die Fortsetzung der von Marx angerissenen Probleme brauchbar sein können.

Jannis Milios und Dimitris P. Sotiropoulos wenden sich schließlich älteren und neueren Ansätzen der Imperialismustheorie zu. Sie zeigen auf, dass sowohl die verschiedenen imperialismustheoretischen Konzepte als auch deren scheinbare Überwindung in Hardt/ Negris Konstruktion eines „Empire“ sich gleichermaßen einer Verabschiedung des Marxschen Konzeptes des gesellschaftlichen Gesamtkapitals verdanken, das zugunsten einer bloß äußerlichen Beziehung von Staat und Kapital aufgegeben wird. Damit, so ihre These, wird eine wesentliche Dimension der Analyse des Kapitalismus auf Weltebene verfehlt.

Außerhalb des Schwerpunkts beschäftigen sich Klaus Müller und Rudi Schmidt mit den Hintergründen sowohl der Krise Griechenlands als auch der Krise des Euro und der EU.

$$
* * *
$$

Am 24. April fand in Berlin die Mitgliederversammlung der „Vereinigung zur Kritik der politischen Ökonomie e.V.“ statt. Die PROKLA wird von der Vereinigung herausgegeben, auf der jährlichen Mitgliederversammlung werden Redaktion 
und Redaktionsbeirat gewählt. Aufgrund beruflicher und familiärer Belastungen kandidierte Christina Deckwirth leider nicht mehr für die Redaktion. Neu in die Redaktion gewählt wurden Silke van Dyk und Stefan Schmalz.
Auch im Redaktionsbeirat gab es Veränderungen: Unser langjähriges Vereinsmitglied Bodo Zeuner kandidierte aus persönlichen Gründen leider nicht mehr, neu in den Beirat gewählt wurde Ulf Kadritzke.

\section{PROKLA 160: Kulturkämpfe (September 2010)}

Es mehren sich die Anzeichen eines neuen Kulturkampfes für die Wiederbelebung „bürgerlicher" Vorstellungen von Freiheit und Gleichheit, gegen die sozialen Rechte und Alltagspraktiken der „Unterschichten“. In Verlängerung der wissenschaftlichpublizistischen Aktivitäten von Vertretern der jüngeren (allen voran Paul Nolte) wie älteren Generation (an vorderer Front etwa Arnulf Baring) hat der Fernsehphilosoph Peter Sloterdijk ein „bürgerliches Manifest“ veröffentlicht, das die politische Weltanschauung und die gesellschaftlichen Ordnungsvorstellungen einer selbsternannten deutschen Neobourgeoisie widerspiegelt. Zu den Bausteinen neuen Bürgerlichkeitskults gehören die ressentimentgeladene Polemik auch noch gegen die Restbestände eines Steuerstaates, der die gesellschaftlichen „Leistungsträger“ zum Zwecke der Umverteilung „ausbeuten“ würde; eine offensive Entwertung der stereotyp als unproduktiv und parasitär dargestellten Lebensweisen von - migrantischen wie eingeborenen - Transferempfängerhaushalten, verbunden mit einer aggressiven, auf diese Schichten zielenden gesellschaftlichen Umerziehungsprogrammatik; schließlich eine offen elitäre Attitüde in Verbindung mit einem (nicht immer nur) latenten Antiparlamentarismus. All das ist mehr als nur die intellektuelle Begleitmusik zu „Schwarz-Gelb“. Er zeugt von einem gesellschaftlichen Klimawandel: bürgerliche Distinktion von (sub)proletarischer Lebensführung ist wieder gefragt. Dieses neue Selbstbewusstsein der bourgeois erleben wir nicht nur im Feuilleton der einschlägigen Medien. Es drückt sich auch in den immer häufigeren Fällen fristloser Entlassung langjährig verdienter Angestellter aufgrund von (angeblichen) Bagatellschädigungen ihrer Arbeitgeber aus, die von der Renaissance einer radikalen, rechtlich gedeckten Eigentumsideologie künden. Und es offenbart sich etwa in der Hamburger Bürgerbewegung gegen die - von bürgerlichen Parteien - beabsichtigte Verlängerung des gemeinsamen Schulbesuchs von Kindern aus heterogenen Sozialmilieus. In der PROKLA wollen wir diese Prozesse analysieren, ideologiekritisch nach den zugrundeliegenden Deutungsmustern und Wertprogrammatiken fragen, dem Phänomen neu-bürgerlicher Kulturkämpfe in unterschiedlichen gesellschaftlichen Lebensbereichen nachgehen und nicht zuletzt die Optionen und Realitäten widerständiger sozialer Praktiken gegen die Kultur neuer Bürgerlichkeit untersuchen. 